\title{
Charged Pion Polarizability From the Lattice
}

\author{
Walter Wilcox ${ }^{\mathrm{a}}$ \\ ${ }^{a}$ Department of Physics, Baylor University \\ Waco, TX 76798-7316
}

Direct evaluation of charged particle polarizabilities on the lattice is quite difficult. However, a short cut for charged pion polarizability - the Das, Mathur, Okubo Sum Rule - can readily be calculated using lattice techniques. A phenomenological model has been developed to fit the time behavior of the propagators in this expression. Numerical systematics are discussed and some preliminary results are presented.

\section{Introduction}

External field methods have been used previously to measure neutral particle polarizabilities in the context of lattice QCD[1]. However, there have been no investigations of charged hadron polarizabilities using the lattice techniques. This is unfortunate since there is now a growing body of experimental information on this subject. Of course, external field methods would be very difficult or impossible to use in the case of charged hadrons because charged particles accelerate in an electric field. The evaluation of charged particle polarizabilities can be done on the lattice by measuring a Compton scattering coefficient [2]. However, the direct evaluation is actually quite difficult because of the many diagrams involved.

There is a way around this problem if one is willing to work in the exact chiral limit. Let us examine a sum rule for pion polarizability and see how readily it can be evaluated on the lattice. Conventional wisdom is that charged pion polarizability involves cancellation of large numbers, so may be difficult to simulate.

\section{DMO Sum Rule and Model}

The Das, Mathur, Okubo Sum rule for the charged pion polarizability, derived from current algebra in the chiral limit is [3]:

$$
\begin{aligned}
\alpha_{E}= & \alpha \frac{<r^{2}>}{3 m_{\pi}} \\
& +\frac{\alpha}{m_{\pi} F_{\pi}^{2}} \int_{4 m_{\pi}^{2}}^{\infty} \frac{d s^{2}}{s^{4}}\left(\rho_{A}\left(s^{2}\right)-\rho_{V}\left(s^{2}\right)\right) .
\end{aligned}
$$

This employs only mesonic two-point functions, which are easily calculable on the lattice. The spectral integrals can be measured using a combination of numerical techniques, actually developed other physics contexts (the "volume method" [- $[$ and "Fourier reinforcement" [5]), which results in a very good signal. The value of the term proportional to the pion squared charge radius, $\left\langle r^{2}\right\rangle$, and the pion decay constant, $F_{\pi}$, are taken from experiment. One can change the spectral integral above into a lattice space-time integral through the use of (the momentum transfer is purely spatial, $\left.q_{\mu}=(0, \vec{q})\right)$

$$
\begin{aligned}
\left.\frac{d}{d \vec{q}^{2}}\left(\Delta_{00}^{V}\left(\vec{q}^{2}\right)-\tilde{\Delta}_{00}^{A}\left(\vec{q}^{2}\right)\right)\right|_{\vec{q}^{2}=0} & = \\
& \int_{4 m_{\pi}^{2}}^{\infty} \frac{d s^{2}}{s^{4}}\left(\rho_{A}\left(s^{2}\right)-\rho_{V}\left(s^{2}\right)\right),
\end{aligned}
$$

where in Euclidean infinite space $\left(V_{0}(x) \equiv\right.$ $\left.\bar{d}(x) \gamma_{0} u(x)\right)$

$$
\begin{aligned}
& \Delta_{00}^{V}\left(\vec{q}^{2}\right)= \\
& \quad \int d^{4} x e^{-i q \cdot x}<0\left|T\left(V_{0}(x) V_{0}^{\dagger}(0)\right)\right| 0>.
\end{aligned}
$$

(The subtracted axial propagator, $\tilde{\Delta}_{00}^{A}\left(\vec{q}^{2}\right)$, is similar to Eq.(3) but has the pion pole removed.) The left-hand side of Eq.(2) is formed by taking a numerical momentum derivative of the lattice data.

There will be new sources of systematic uncertainty in the lattice evaluation of sum rules. In this case the above shows that one must perform a numerical momentum derivative. In addition, 
the time integral can be performed in either a discrete or continuous sense, with attendant uncertainty. One can simply apply Simpson's rule in the discrete case. The continuous case demands some way of interpolating between the lattice time points. For this purpose, let us consider the standard lattice point-to-point charge density correlator:

$$
\sum_{\vec{x}} e^{-i \vec{p} \cdot \vec{x}}<0\left|T\left(V_{0}(x) V_{0}^{\dagger}(0)\right)\right| 0>.
$$

One may show that (all masses and distances dimensionless) this reduces to

$$
\sum_{\vec{x}} e^{-i \vec{p} \cdot \vec{x}} \operatorname{Tr}\left[S(x, 0) \gamma_{4} \gamma_{5} S^{\dagger}(x, 0) \gamma_{4} \gamma_{5}\right],
$$

where $S(x, y)$ is the quark propagator and the trace is over color and Dirac spaces. Use the free quark propagator (coordinate gauge; diagonal to this order in color space):

$S(x, 0)=\frac{1}{2 \pi^{2}} \frac{\gamma \cdot x}{x^{4}}+\frac{1}{(2 \pi)^{2}} \frac{m_{q}}{x^{2}}+\cdots$.

Make the replacement $\sum_{x} \rightarrow \int d^{3} x$ and define

$G_{00}^{V}(t, \vec{p}) \equiv \int d^{3} x e^{-i \vec{p} \cdot \vec{x}} \frac{t^{2}-r^{2}}{\left(r^{2}+t^{2}\right)^{4}}$.

The next term is of order $m_{q}^{2}$ and is negligible in our phenomenological fits. Do the angular integrals $(p \equiv|\vec{p}|)$ :

$G_{00}^{V}(t, \vec{p})=$

$\frac{12}{\pi^{3} p} \int_{0}^{\infty} d r r \sin (p r)\left\{\frac{2 t^{2}}{\left(r^{2}+t^{2}\right)^{4}}-\frac{1}{\left(r^{2}+t^{2}\right)^{3}}\right\}$.

Actually, what one wants in this case is the derivative of the above with respect to $p^{2}$ (see Eq.(2) above). I prefer to form the same numerical derivative in the phenomenological model as is necessary on the lattice. Thus, in order to compare with the lattice data, consider

$$
\begin{aligned}
\mathcal{G}_{00}^{V}( & t, \vec{p}) \equiv \frac{G_{00}^{V}(t, \vec{p})-G_{00}^{V}(t, 0)}{p^{2}} \\
= & \frac{12}{\pi^{3} p^{2}} \int_{0}^{\infty} d r r\left[\frac{\sin (p r)}{p}-r\right] \\
& \times\left\{\frac{2 t^{2}}{\left(r^{2}+t^{2}\right)^{4}}-\frac{1}{\left(r^{2}+t^{2}\right)^{3}}\right\} .
\end{aligned}
$$

To this order the same functional form holds for the axial propagator as well.

There are two modifications to this function that are necessary before one compares to the lattice data. First, it is clear that the above expressions have an ultraviolet infinity associated with the $r=0$ lower limit. This infinity can be controlled, as the lattice itself controls it, by putting in a short distance cutoff. So, replace the lower limit above by $r_{0}>0$, which becomes a parameter in the fits. Call this modified function $\mathcal{G}_{00}^{V}\left(t, \vec{p}, r_{0}\right)$. Second, put in a continuum threshold, $s_{0}$, to control the onset of excited states in the spectral density. One can easily show that the resulting function is now given by $(t>0$ understood)

$$
\begin{aligned}
& \hat{\mathcal{G}}_{00}^{V}\left(t, p, r_{0}, s_{0}\right) \equiv \\
& \quad \zeta \int_{s_{0}}^{\infty} d s \int_{-\infty}^{\infty} \frac{d u}{2 \pi} e^{i u s} \mathcal{G}_{00}^{V}\left(t+i u, \vec{p}, r_{0}\right) .
\end{aligned}
$$

A third parameter, $\zeta$, has been added in Eq.(8) in order to account for lattice anisotropy at small lattice times as in Ref.[6]. One can analytically do the two integrals in Eq.(8); the remaining oscillating radial integral is done numerically. One must also apply vector and axial vector renormalization constants to the lattice data before comparing to Eq.(8). In addition, add to Eq.(8) a function

$$
\lambda^{2} e^{-m_{V, A} t},
$$

to fit the lowest pole mass in the vector (rho meson) or axial vector (A1 meson) cases. These mass values are fixed from previous independent lattice measurements, so all told there are 4 parameters in the model: $\zeta, \lambda, s_{0}$ and $r_{0}$.

Using Wilson fermions at $\beta=6.0$ on a $16^{3} \times 24$ lattice, one has $p_{\min }=\frac{\pi}{8}$, and this value is used in both the numerical lattice derivative as well as the phenomenological model. I have not yet done the chiral extrapolation in quark mass, but the results from Eq.(11) when plotted as a function of quark mass are extremely flat.

The fit vector correlator (unnormalized) is shown on a $\log _{10}$ scale in Fig. 1 at $\kappa=0.152$. It is the area under this curve which is of interest here. The time integrals are very sharply peaked near $t=0$. The numerical quality of the vector time data is seen to be quite good. 
Figure 1. Vector correlator at $\kappa=0.152$. The dashed line is the fit from the free quark model.

My preliminary result (at $\kappa=.154$ ) is (using the lattice scale from Ref.[7])

$$
\alpha_{E}=1.7(2.1)(.8) \times 10^{-4} \mathrm{fm}^{3}
$$

where the first number in parenthesis is the systematic uncertainty in the time integral, the second is statistical. The systematic uncertainty is defined to be half of the difference between the discrete (Simpson's rule - smaller value) and continuous (free quark model - larger value) evaluations for $\alpha_{E}$. The above is to be compared with the experimental result (from MARK-II data [8]),

$$
\alpha_{E}^{e x p}=2.2(1.6) \times 10^{-4} \mathrm{fm}^{3}
$$

and the result from second order chiral perturbation theory 9],

$$
\alpha_{E}^{\text {chiral }}=2.4(.5) \times 10^{-4} \mathrm{fm}^{3} .
$$

So what one gets is surprisingly good, at least compared to experiment. Warning: other sources of systematic error (momentum derivative, lattice scale) have not yet been evaluated.

\section{Summary and Remarks}

The sum rule method of extracting charged pion polarizability from lattice data has been examined. We have seen that the systematic uncertainty in the time integral is larger than the statistical uncertainty, but still reasonable compared to experiment.

To reduce this systematic uncertainty, the situation here demands a fine mesh of lattice points in the time direction. Coarse lattices are not useful in the case of such sum rules. However, time asymmetric lattices with a small time spacing (but not too small to get into the asymptotic regime) could possibly be very useful.

Pion polarizability scales like $a^{3}$, and so is extremely sensitive to the lattice scale. When the experimental situation is improved, it could easily become one of the most sensitive tests of QCD.

\section{Acknowledgements}

This work is supported in part by NSF Grant No 9401068 and the National Center for Supercomputing Applications. The author would like to thank J. Vasut and B. Lepore for help with the free quark model integral.

\section{REFERENCES}

1. H. R. Fiebig, W. Wilcox, and R. M. Woloshyn, Nucl. Phys. B324 (1989) 47.

2. W. Wilcox, "Lattice Charge Overlap II: Aspects of Charged Pion Polarizability", BU/HEPP/96-02 (hep-lat/9606019).

3. T. Das, V. S. Mathur and S. Okubo, Phys. Rev. Lett. 19 (1967) 859.

4. Y. Kuramashi et. al., Phys. Rev. Lett. 71 (1993) 2387; W. Wilcox, S. Trendafilov and E. Mendel, Nucl. Phys. B (Proc. Suppl.) 42 (1995) 557.

5. W. Wilcox and B. Andersen-Pugh, Nucl. Phys. B (Proc. Suppl.) 34 (1994) 393.

6. D. B. Leinweber, Phys. Rev. D51 (1995) 6369.

7. K. F. Liu, S. J. Dong, T. Draper and W. Wilcox, Phys. Rev. Lett. 74 (1995) 2172.

8. D. Babusci et. al., Phys. Lett. B277 (1992) 158. 
9. U. Bürgi, "Charged Pion-Pair Production and Pion Polarizabilities to Two Loops, BUTP-96/02 (hep-ph/9602429). 